\title{
Modeling and Performance of a 100-Element pHEMT Grid Amplifier
}

\author{
Michael P. De Lisio, Member, IEEE, Scott W. Duncan, Member, IEEE, Der-Wei Tu, Cheh-Ming Liu, \\ Alina Moussessian, James J. Rosenberg, and David B. Rutledge
}

\begin{abstract}
A 100 -element hybrid grid amplifier has been fabricated. The active devices in the grid are custom-made pseudomorphic high electron mobility transistor (pHEMT) differentialpair chips. We present a model for gain analysis and compare measurements with theory. The grid includes stabilizing resistors in the gate. Measurements show the grid has a peak gain of 10 dB when tuned for $10 \mathrm{GHz}$ and a gain of $12 \mathrm{~dB}$ when tuned for $9 \mathrm{GHz}$. The maximum $3-\mathrm{dB}$ bandwidth is $15 \%$ at $9 \mathrm{GHz}$. The minimum noise figure is $3 \mathrm{~dB}$. The maximum saturated output power is $3.7 \mathrm{~W}$, with a peak power-added efficiency of $12 \%$. These results are a significant improvement over previous grid amplifiers based on heterojumction bipolar transistors (HBT's).
\end{abstract}

\section{INTRODUCTION}

Q UASI-OPTICAL amplifiers combine the output powers of many solid-state devices in free space, eliminating the losses associated with waveguide or transmissionline combiners. The first quasi-optical amplifier was a 25 element grid amplifier [1]. A grid amplifier is an array of closely-spaced differential pairs of transistors. Fig. 1 shows the approach. A horizontally polarized input beam excites rf currents on the input leads of the grid. This drives the transistor pair in the differential mode. Currents on the output leads produce a vertically polarized output beam. Metal-strip polarizers provide independent tuning of the input and output circuits. Other types of quasi-optical amplifiers using patch antennas [2]-[4], back-to-back integrated horn antennas [5], [6], folded slot antennas [7], and probe antennas [8] have been demonstrated. The largest number of devices have been incorporated in a 100 -element HBT grid amplifier [9].

Recently, pHEMT technology has developed rapidly. Millimeter-wave pHEMT's are capable of high gain, high output powers, and low noise figure, making them the device of choice for high-frequency applications [10]-[12]. Here we

Manuscript received April 10, 1995, revised September 13, 1995. This research was supported by the Army Research Office and Lockheed Martin Laboratories. M. P. De Lisio held NSF and AASERT fellowships.

M. P. De Lisio is with the Department of Electrical Engineering, University of Hawaii-Mānoa, Honolulu, HI 96822 USA.

S. W. Duncan is with Sanders, a Lockheed Martin Company, Nashua, NH 03061 USA.

D.-W. Tu is with the Laboratory for Physical Sciences, University of Maryland, College Park, MD 20783. USA.

C.-M. Liu is with the Rockwell Science Center, Rockwell International Corp., Thousand Oaks, CA 91385 USA.

A. Moussessian and D. B. Rutledge are with the Department of Electrical Engineering, California Institute of Technology, Pasadena, CA 91125 USA.

J. J. Rosenberg is with the Department of Engineering, Harvey Mudd College, Claremont, CA 91711 USA.

Publisher Item Identifier S 0018-9480(96)08479-7.

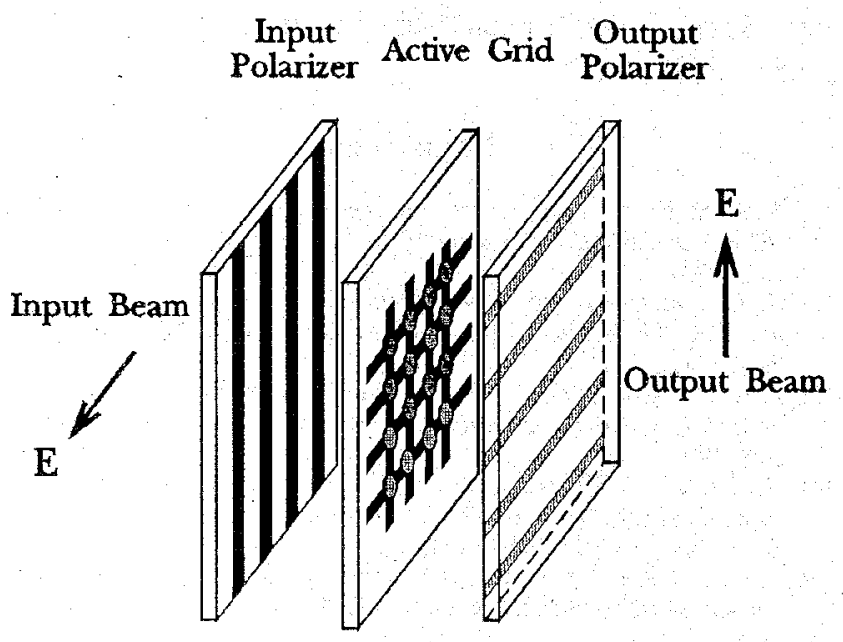

Fig. 1. A grid amplifier. A horizontally polarized input beam is incident from the left. The output beam is vertically polarized and is tadiated to the right. The polarizers independently tune the output and input circuits.

report on the modeling and performance of a 100 -element $X$-band grid amplifier using pHEMT differential-pairs. A photograph of the assembled grid is shown in Fig. 2.

\section{DEVICE DESCRIPTION}

The differential-pair chips were fabricated by Lockheed Martin Laboratories. The active devices are $0.1-\mu \mathrm{m} \mathrm{Al}$ GaAs/InGaAs/GaAs pHEMT's. The total gate width per transistor is $100 \mu \mathrm{m}$ distributed among four fingers. The gates were defined by an electron beam direct write system and a bi-level resist. The pHEMT epitaxial structure is a double-doped heterostructure optimized for power. Further details about the devices can be found in [10].

The differential pair chip layout is shown in Fig. 3. The sources of two pHEMT's are tied together to form a differential pair. Unlike the HBT's in previous grids $[9,14]$, the control terminal can be externally biased. The $1-\mathrm{k} \Omega$ resistor allows this gate control bias to pass from cell to cell. This is possible because the gate draws very little bias current The $0.5-\mathrm{pF}$ capacitor and $2-k \Omega$ resistor are feedback elements to stabilize the pHEMT's. The two $100-\Omega$ source resistors are intended to reduce common-mode gain. These resistors should not effect the differential-mode performance, but will reduce the overall efficiency of the grid amplifier. 


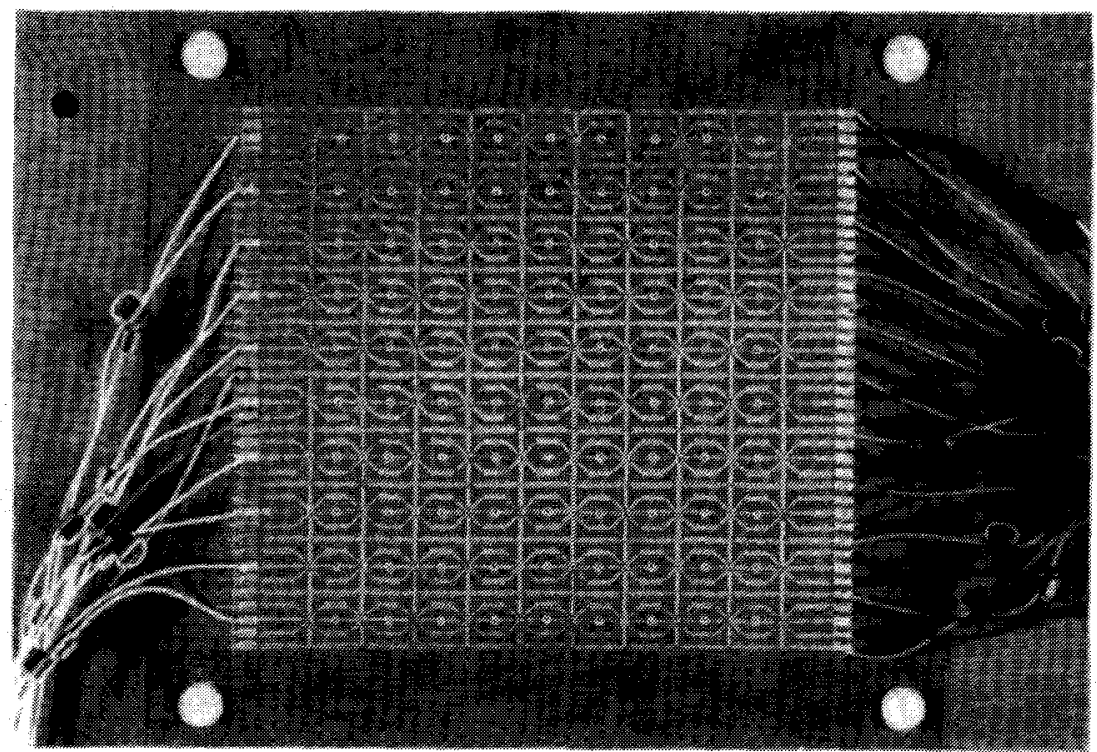

Fig. 2. Photograph of the amplifier grid. The grid is a $10 \times 10$ array of pHEMT differential pairs. The active area of the grid is $7.3 \mathrm{~cm}$ on a side

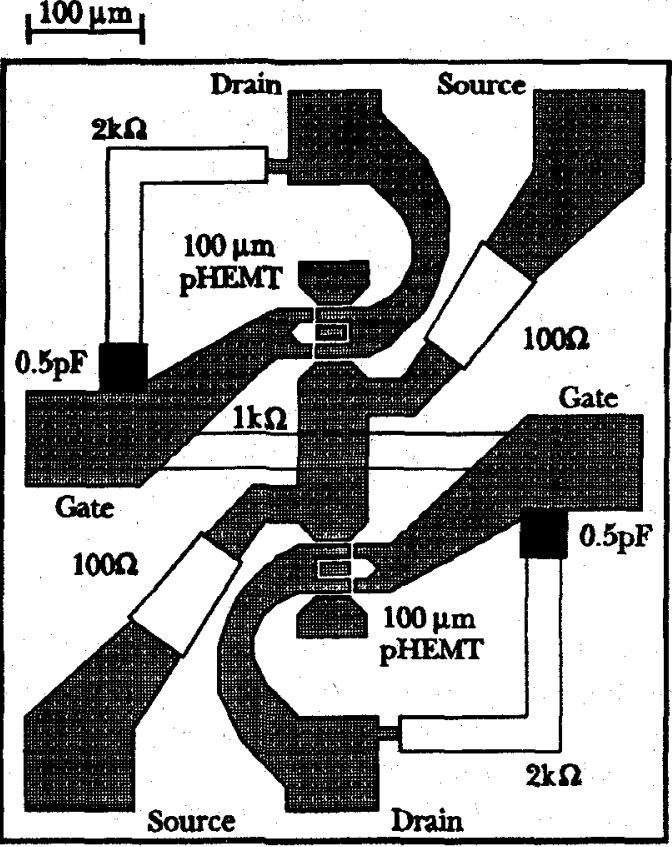

Fig. 3. The differential pair chip layout.

\section{GRID AMPLIFIER MODELling}

An important advantage of grid amplifiers is that the unit cell primarily determines the driving-point impedances seen by the device, while the power scales with the grid area. This allows one to optimize for gain and noise figure by the choice of the unit cell and to independently select the grid size to meet the total power requirement. Previous grid amplifiers [1], [9] were designed empirically. We have developed a model for the grid amplifier that predicts its performance [13], [14].

The unit cell is shown in Fig. 4. The cell size is $7.3 \mathrm{~mm}$ on a side. The input beam is coupled to the gates of the transistors through the horizontal gate leads. The output beam

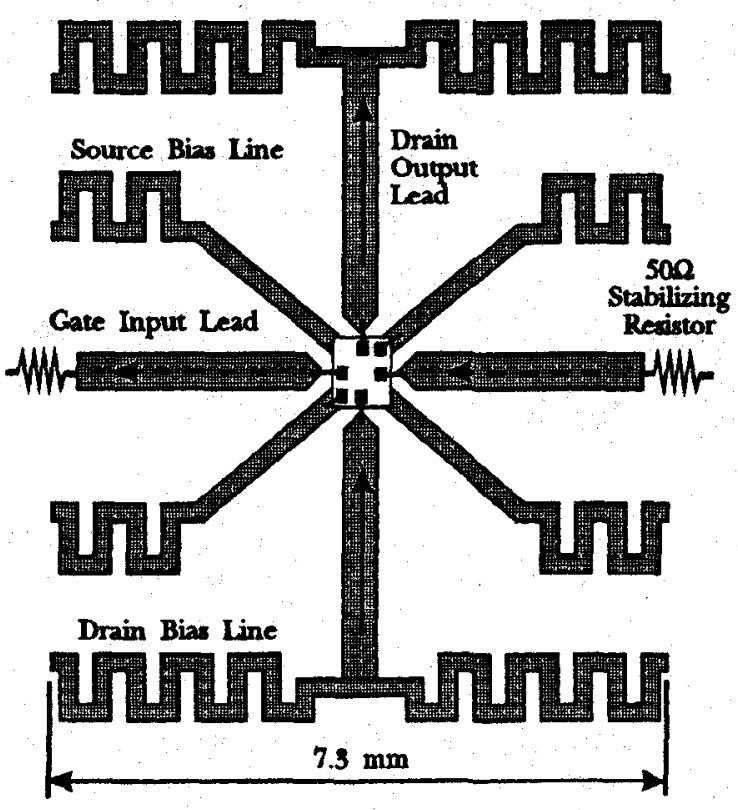

Fig. 4. The grid amplifier unit cell. The width of the input and output leads is $0.4 \mathrm{~mm}$. The width of the meandering bias lines is $0.2 \mathrm{~mm}$. Arrows indicate the directions of if currents.

is re-radiated from the vertical drain leads. Bias to the drain and source is provided by the thin meandering lines. Gate bias is provided along the horizontal gate leads. The resistors in the gate leads suppress common-mode oscillations and will be discussed later.

The assembled grid amplifier tuned for $10 \mathrm{GHz}$ is shown in Fig. 5(a). The grid and polarizers are fabricated on Rogers Duroid boards with a relative dielectric constant of 2.2. The output tuner is a Duroid board with $\epsilon_{r}=10.5$. A simple transmission-line model for the grid amplifier is shown in Fig. 5(b). For convenience, one-half of the unit cell is used in the analysis, with the result that the characteristic impedances 


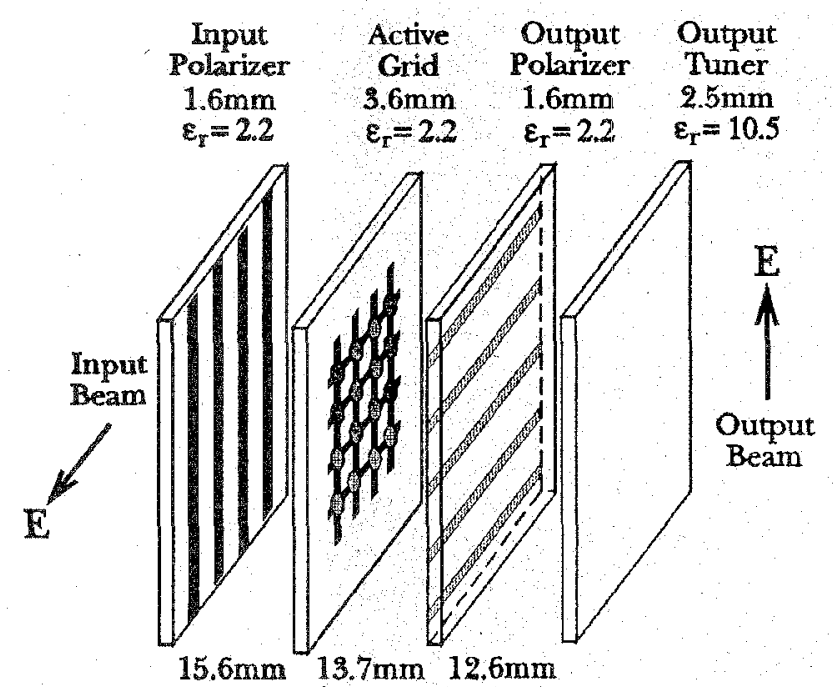

(a)

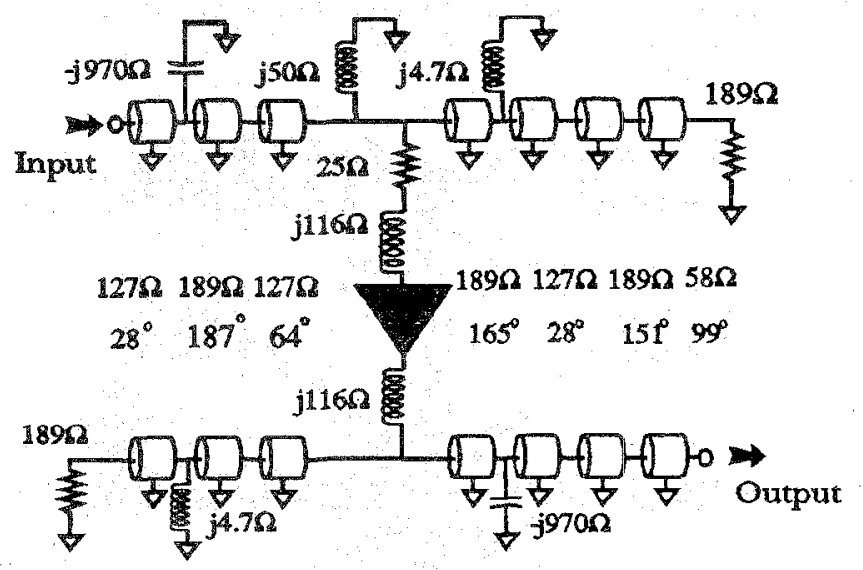

(b)

Fig. 5. The assembled grid amplifier (a). The polarizer and tuning-slab positions are adjusted for optimum gain at $10 \mathrm{GHz}$. (b) Transmission-line equivalent circuit for the grid amplifier at $10 \mathrm{GHz}$.

are one-half of their usual values. Free-space, then, is represented by $189-\Omega$ resistors. The various substrates and air gaps appear as transmission lines. The metal-strip polarizers appear in shunt, either as low-impedance inductors or high-impedance capacitors, depending on the polarization. The input and output leads of the grid are modeled as inductors. The meandering drain and source bias lines are modeled as a shunt inductance at the input surface of the grid.

Numerical values for the radiating lead and polarizer reactances are computed by first employing the method of moments to approximate the surface current distribution. We use the point-matching technique with the subsectional basis functions being rectangular for the inductive elements and raised cosines for the capacitive elements [15]. Once an estimate for the surface current has been obtained, the impedance is calculated using the induced emf technique. The numerical value of the reactance associated with the meandering bias lines is empirically determined with the aid of HewlettPackard's High Frequency Structure Simulator (HFSS).

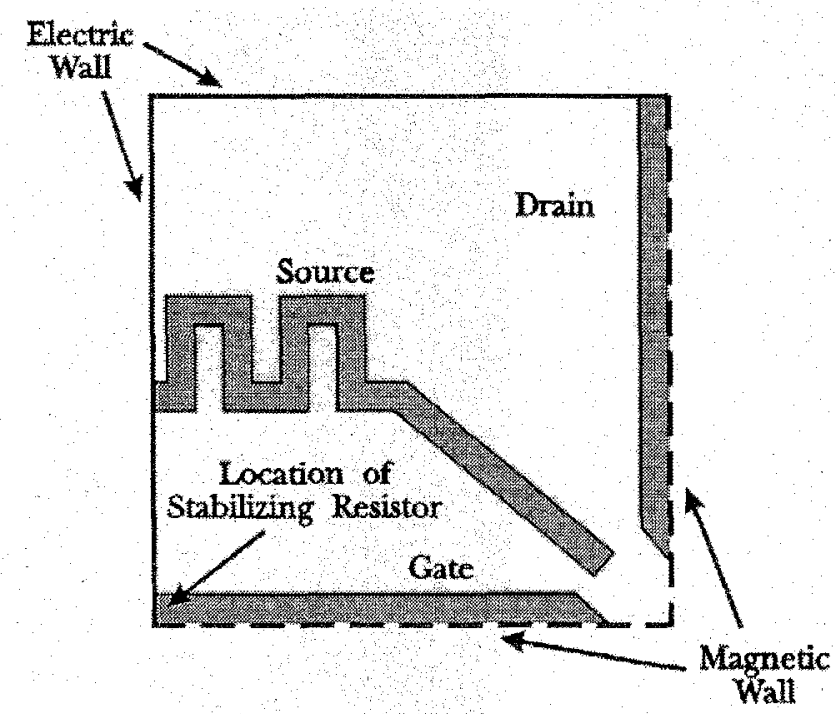

(a)

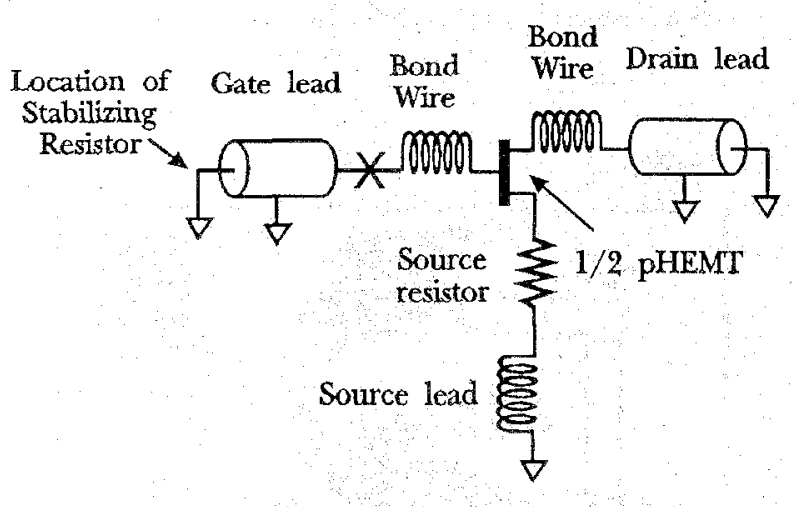

(b)

Fig. 6. Quarter cell (a). The boundary conditions are imposed by grid symmetry. (b) Equivalent circuit model for common-mode oscillation. The $x$ marks the spot where a circulator is added to determine the oscillation frequency.

Initially, the grid suffered from $8-9 \mathrm{GHz}$ oscillations, depending on the bias. The onset of the oscillations was at a bias current of $9 \mathrm{~mA}$ per transistor-well below the transistor's usual operating current. The gain of the amplifier at the onset of oscillation was $3 \mathrm{~dB}$. These oscillations were insensitive to polarizer position and substrate thickness, leading us to conclude that the oscillation was a common-mode oscillation like the one described in [14]. Our model for the commonmode oscillations is shown in Fig: 6. We use this model to predict the stability of the grid. Using the approach of Martinez and Compton [16], we insert a circulator at the node marked $x$. The circular function, $C$, is plotted in Fig, 7, $C$ can be thought of as a measure of the open-loop gain of the circuit. This circuit is unstable at $8.9 \mathrm{GHz}$ because the magnitude of $C$ is greater than unity and its phase is zero. This is close to where the actual oscillations occurred.

To suppress these oscillations, we placed $50-\Omega$ resistors in the gate lead, midway between transistor pairs. With these resistors in place, the magnitude of $C$ is less than unity, so the grid should be stable. After adding the resistors, the grid could be biased to a current of over $15 \mathrm{~mA}$ per transistor without 


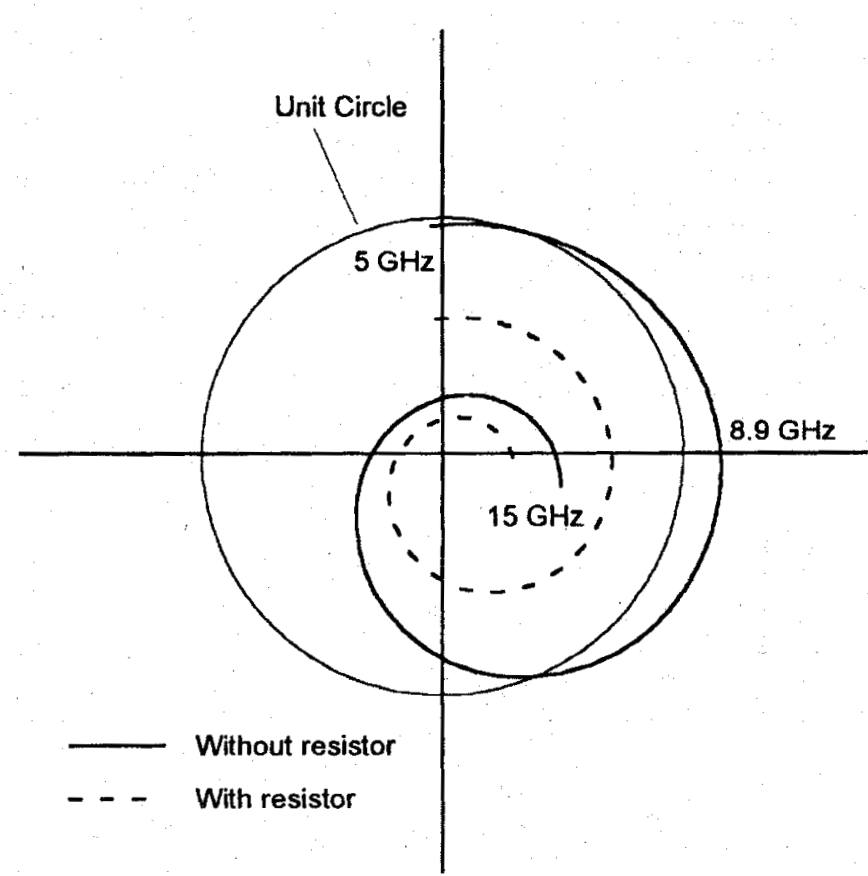

Fig. 7. Circular function of the grid amplifier. Without the stabilization resistors, the grid is unstable at $8.9 \mathrm{GHz}$. The stabilized amplifier has a gain margin of $3 \mathrm{~dB}$.

oscillations. Our model indicates that the resistors degrade the gain by $1.5 \mathrm{~dB}$ and the noise figure by $0.8 \mathrm{~dB}$.

\section{GAIN AND TUNING}

The small-signal gain of the grid was measured by placing the grid in the far field of two cross-polarized horns [9]. The measured gain is shown in Fig. 8(a). The peak gain is $10 \mathrm{~dB}$ at $10 \mathrm{GHz}$. The $3-\mathrm{dB}$ gain bandwidth is $700 \mathrm{MHz}$. The modeled gain is also plotted, and it agrees well with the measured results. Without bias, the gain of the grid was below $-12 \mathrm{~dB}$ throughout the entire frequency range. The difference between the biased and unbiased gain is greater than $30 \mathrm{~dB}$. Fig. 8(b) shows the gain with the output tuner removed. The positions of the polarizers are unchanged. The gain is $1 \mathrm{~dB}$ less at $10 \mathrm{GHz}$, but the $3-\mathrm{dB}$ bandwidth is increased to $1.3 \mathrm{GHz}$. Again, the modeled and measured gain compare well. To some extent, the operating frequency of grid amplifiers can be tuned by simply adjusting the positions of the polarizers and tuners. Fig. 9 shows the gain with the amplifier re-tuned for $9 \mathrm{GHz}$. The gain is $12 \mathrm{~dB}$ at $9 \mathrm{GHz}$ with a 3-dB bandwidth of 1.3 $\mathrm{GHz}$. This corresponds to a $15 \%$ bandwidth.

Fig. 10 shows the gain with the grid tuned for $10 \mathrm{GHz}$ as a function of bias current for three different grid drain voltages. The current was varied under constant drain voltage by adjusting the gate voltage. The drain current was increased until the common-mode oscillations reappeared. These results show that a further increase in bias current is not likely to increase the amplifier gain significantly.

To further validate the model, we measured the gain as a function of polarizer position. Fig. 11(a) shows the gain

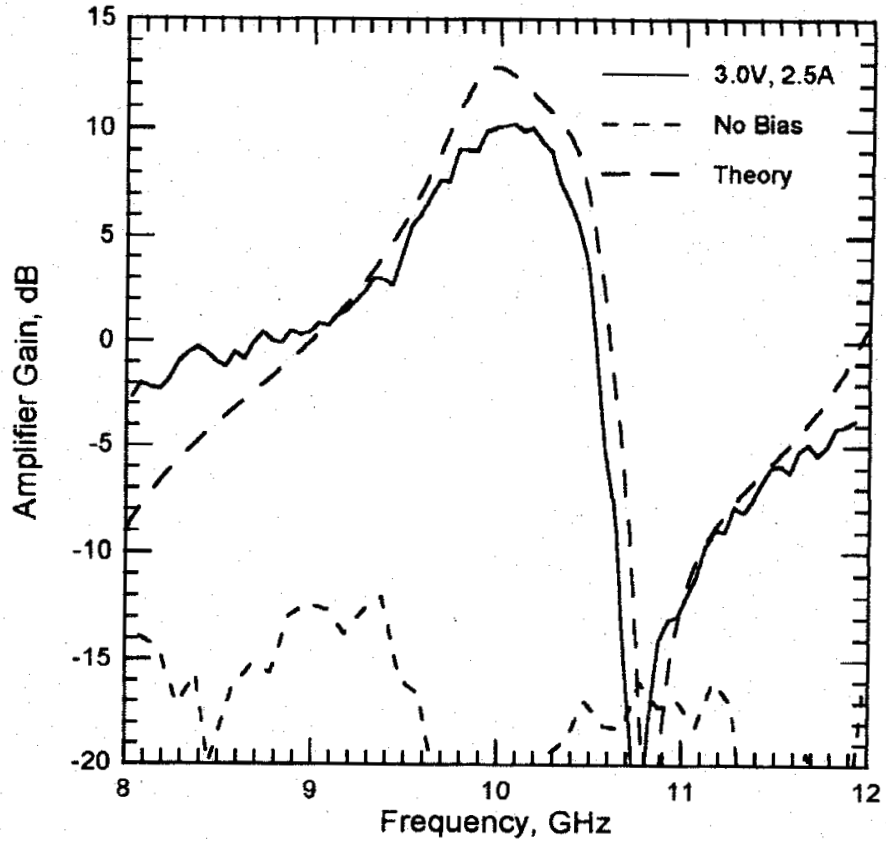

(a)

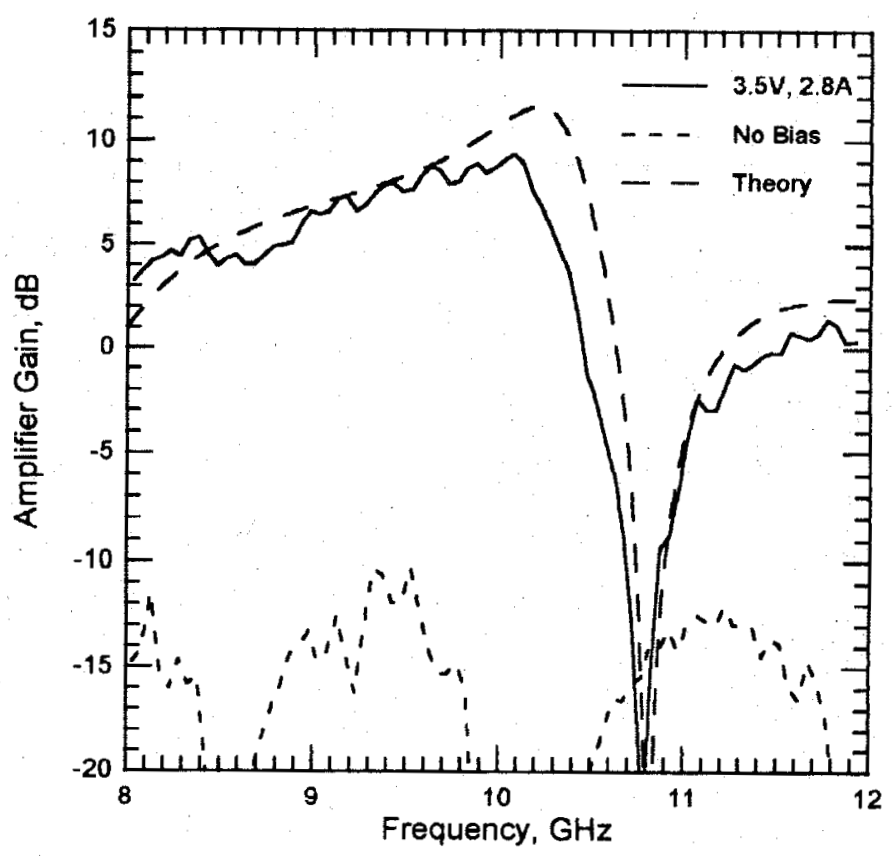

(b)

Fig. 8. Grid amplifier gain versus frequency (a). The polarizer and tuner positions are shown in Fig. 5(a). (b) Gain with output tuner removed.

as a function of input polarizer position. The input polarizer actually tunes the output circuit of the grid. Fig. 11(b) shows the gain as a function of output polarizer position. For convenience, the output tuner was removed for this measurement. The tuning curves agree quite well with the modeled results.

\section{ANGULAR DEPENDENCE}

The amplifier's output $\mathrm{H}$-plane radiation pattern is shown in Fig. 12. This measurement was done by fixing the position of the amplifier and input antenna, and sampling the output pat- 


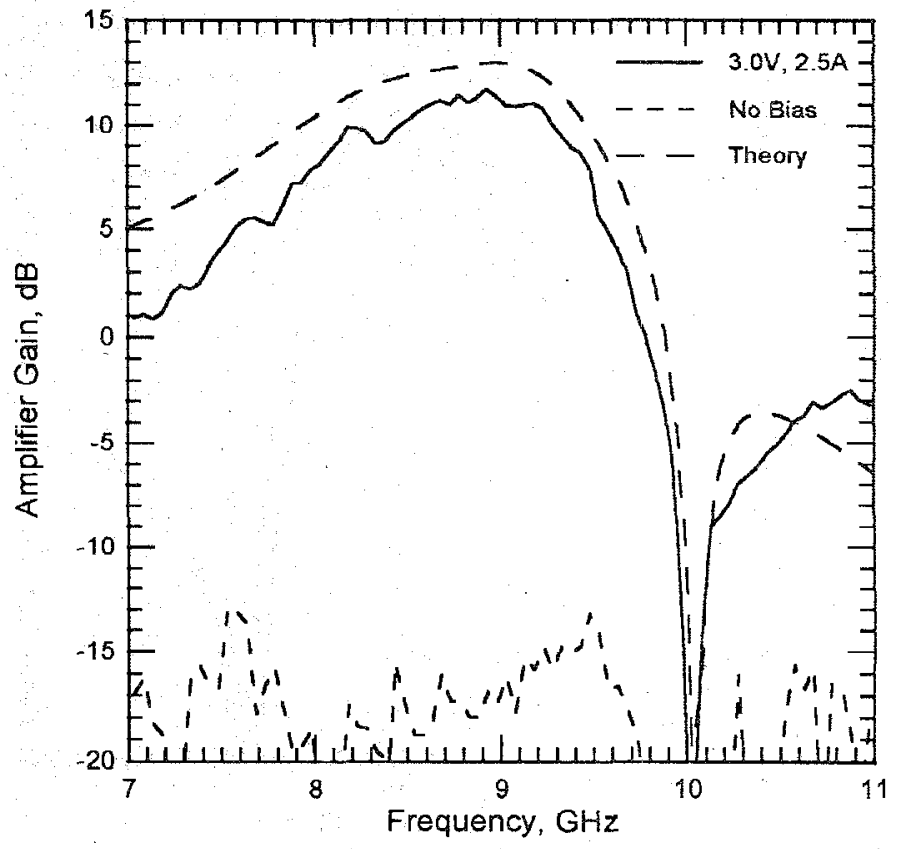

Fig. 9. Amplifier gain with grid tuned for $9 \mathrm{GHz}$.

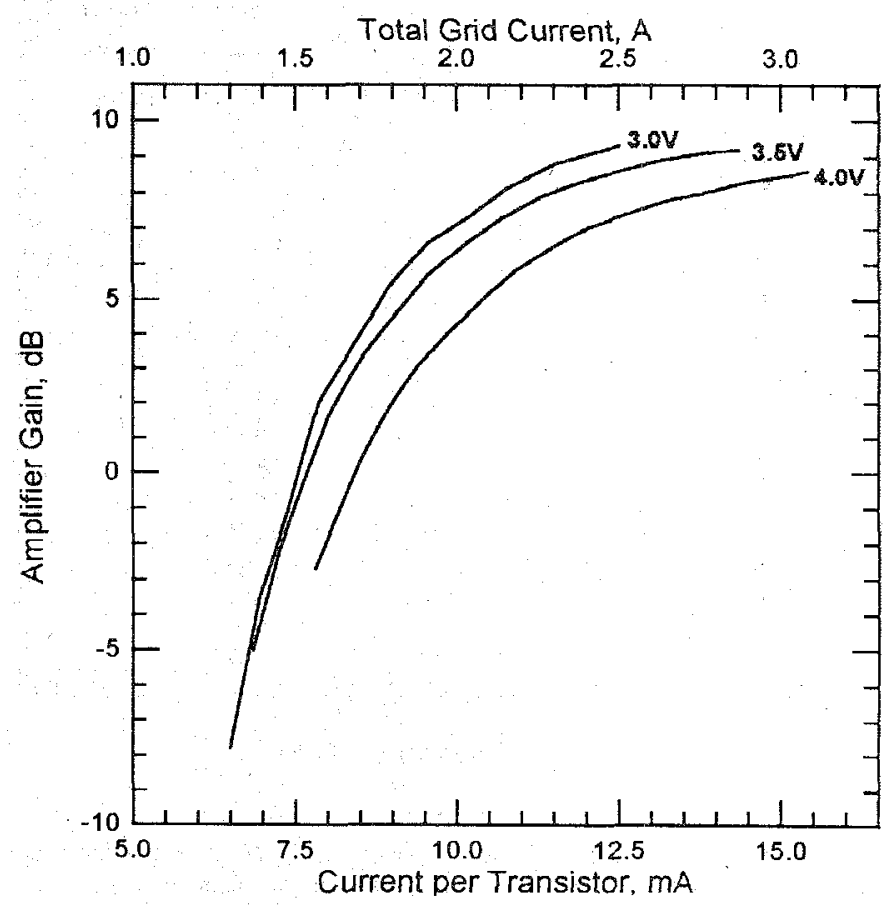

Fig. 10. Amplifier gain at $10 \mathrm{GHz}$ as a function of bias current. The output tuner was not used in this measurement.

tern. For comparison, the theoretical pattern of ten elementary dipoles spaced $7.3 \mathrm{~mm}$ apart is also shown. The agreement between the theory and experiment indicates that the radiation pattern is diffraction-limited.

The grid amplifier is a multimode device, and should be capable of amplifying beams at different angles. We measured the angular dependence of the gain by rotating the grid in between two fixed cross-polarized horns. Fig. 13 shows

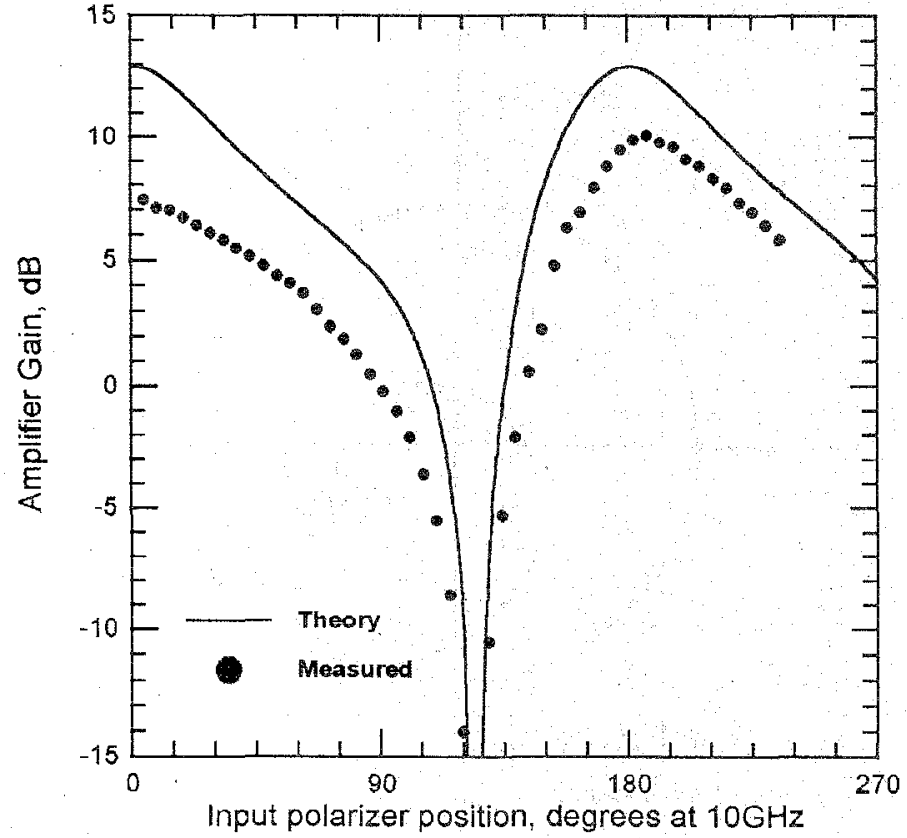

(a)

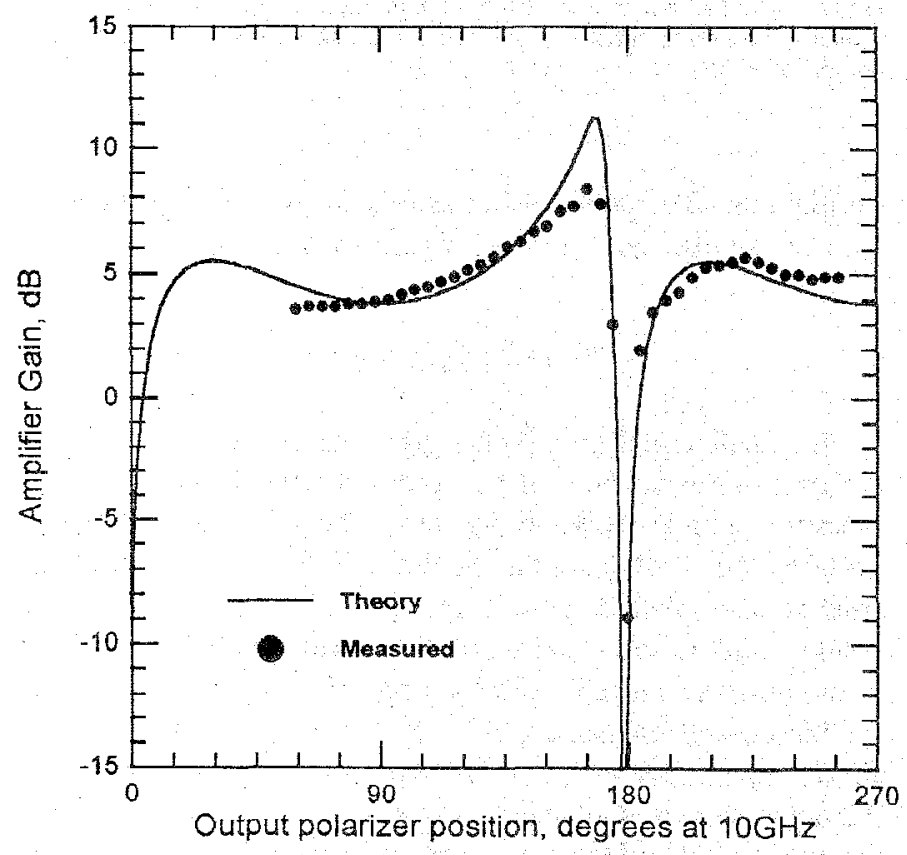

(b)

Fig. 11. Amplifier gain at $10 \mathrm{GHz}$ as a function of input (a) and output (b) polarizer position. For convenience, the output tuner was not used for the measurement in (b).

the results at $10 \mathrm{GHz}$ with the output tuner removed for convenience. For both orientations, the grid will amplify beams with incidence angles up to $25^{\circ}$ with less than a $3-\mathrm{dB}$ drop in gain. The theoretical curves were generated by scaling the lengths of each of the transmission lines in the gain model by $\cos \theta_{i}$, where $\theta_{i}$ is the angle in each material, given by Snell's law. Furthermore, the impedance of each transmission line was multiplied by $\cos \theta_{i}$ for TM waves or $\sec \theta_{i}$ for TE waves. The pattern was then scaled by $a \cos ^{2} \theta$ obliquity factor. 


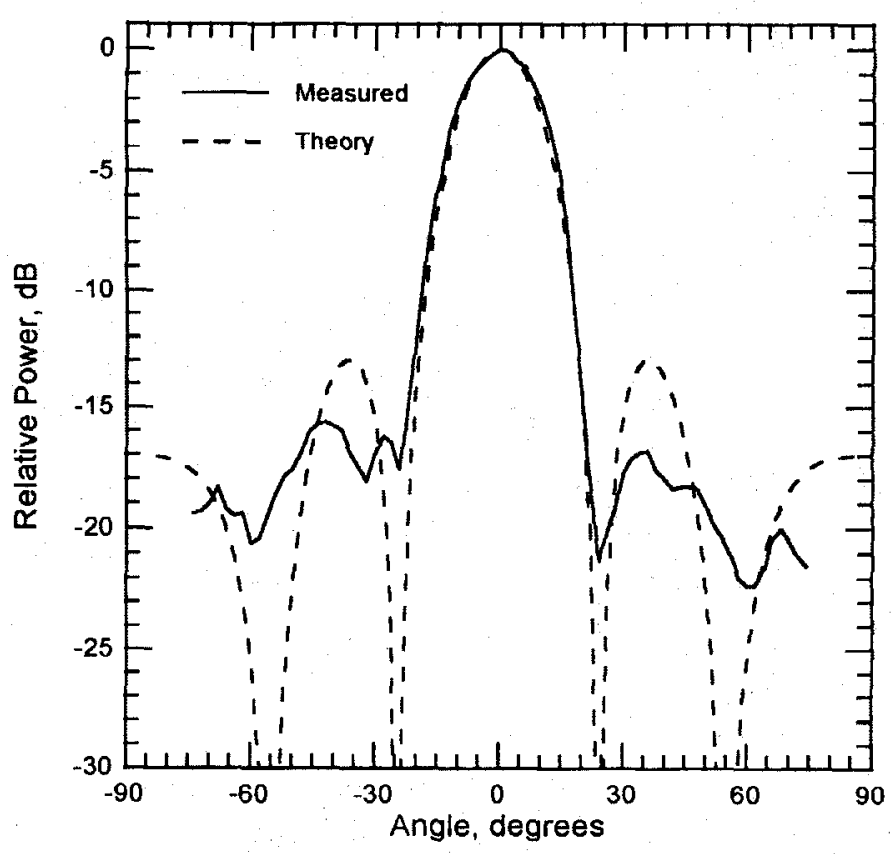

Fig. 12. Output H-plane radiation pattern. The input beam is normal to the grid. Limitations in the measurement setup prohibit measuring the pattern at angles greater than $75^{\circ}$.

Further details can be found in [15]. This theory predicts the pattern well for small angles, but fails at larger angles where the finite area of the grid causes the simple transmission-line model to break down.

The nulls in the patterns are caused by the input polarizer. These nulls disappear when the polarizer is less than a halfwavelength from the active surface. This is shown in Fig. 14. The peak gain of the grid is $3 \mathrm{~dB}$ less with the close polarizer spacing. This may be related to evanescent-wave coupling, as described in [17]. In this configuration, the grid can amplify beams with incident angles up to $30^{\circ}$ with less than a 3-dB gain reduction. These patterns approach the $\cos ^{2} \theta$ obliquity limit.

\section{NOISE}

The noise figure was measured in the far field [9]. We used a Noise Com NC3206 noise source with an Excess Noise Ratio (ENR) of $36 \mathrm{~dB}$ at $10 \mathrm{GHz}$. Fig. 15 shows the result. The minimum noise figure is $3 \mathrm{~dB}$ with $3 \mathrm{~V}$ bias. This is comparable to the noise figure measured in [4] and is a 4- $\mathrm{dB}$ improvement over the HBT grid reported in [9]. The predicted minimum noise figure is given by Fukui's equation [18]

$$
F_{\min }=1+2 \pi f K C_{g s} \sqrt{\frac{R_{s}+R_{g}+R_{s t}}{g_{m}}}
$$

where the transistor source resistance $R_{s}$ is $4.7 \Omega$, the transistor gate resistance $R_{g}$ is $2.4 \Omega$, and the stabilizing gate resistance $R_{s t}$ is $25 \Omega$. Assuming that the material parameter $K$ is $5, F_{\min }=1.6 \mathrm{~dB}$. As shown in the figure, the stabilizing gate resistors degrade $F_{\min }$ by about $1 \mathrm{~dB}$.

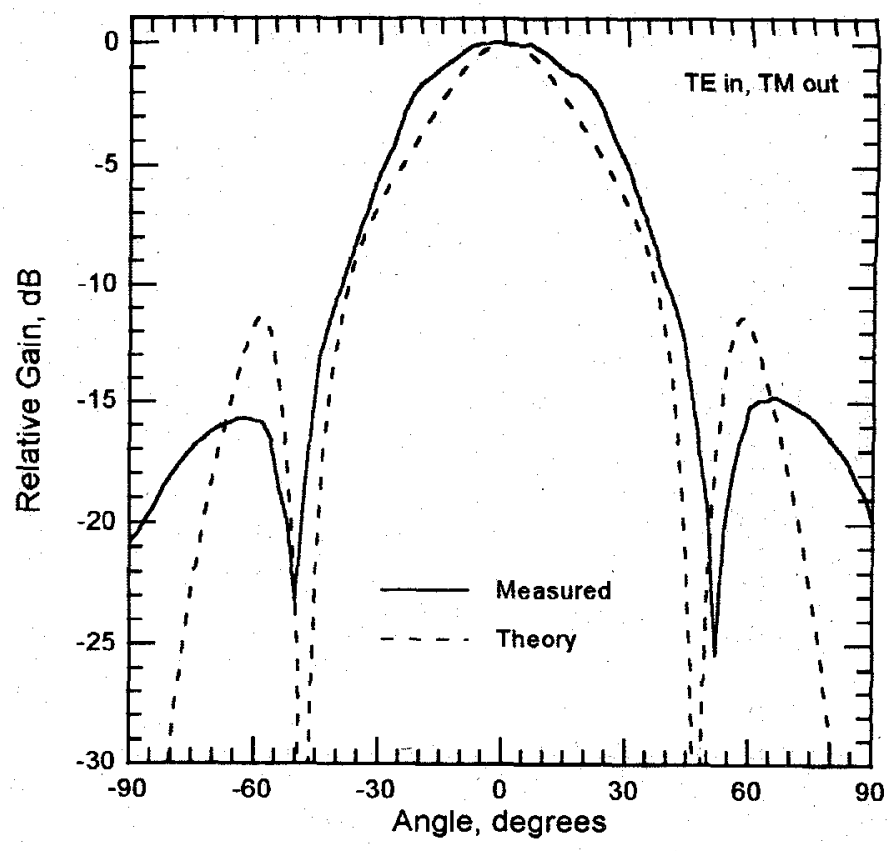

(a)

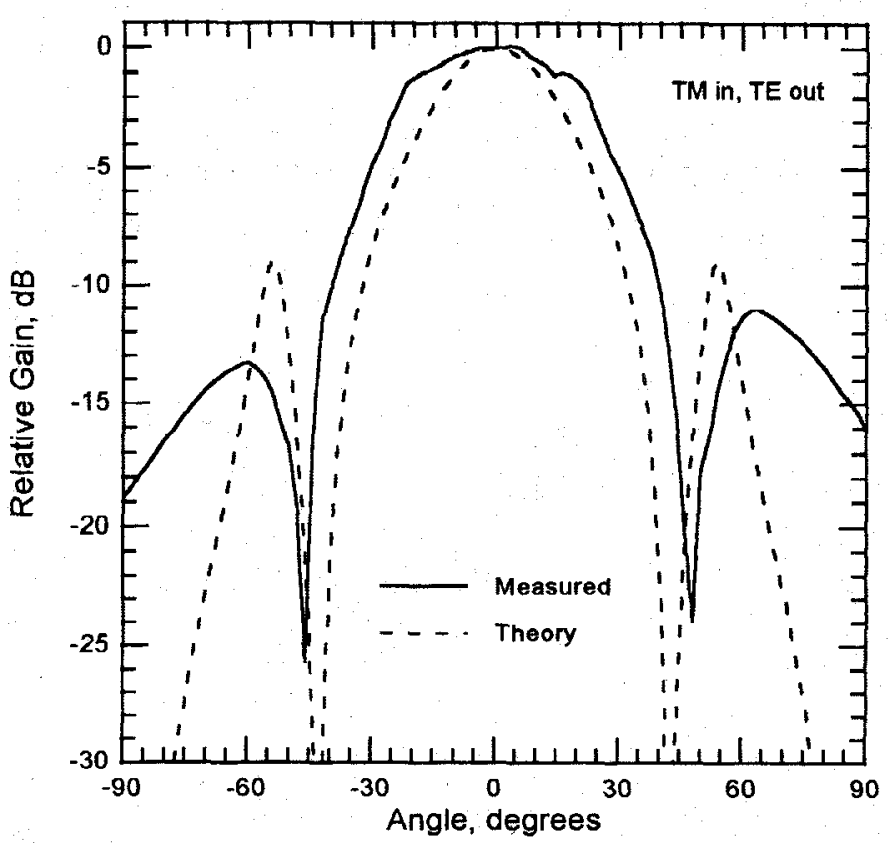

(b)

Fig. 13. Relative amplifier gain at $10 \mathrm{GHz}$ as a function of angle. (a) Input TE polarization, output TM. (b) Input TM polarization, output TE.

\section{POWER}

The power saturation of the grid was also measured in the far field [9]. We used a high-power travelling-wave tube (TWT) amplifier as the source. For this experiment, we tuned the grid amplifier for $9 \mathrm{GHz}$ to match the TWT's output frequency. Fig. 16 shows the result. The peak output power is $3.7 \mathrm{~W}$ at $7 \mathrm{~V}$ bias. This is an 8 -dB improvement over the HBT amplifier grid [9], and the highest reported output power for a quasi-optical amplifier. The dc power supplied to the grid 


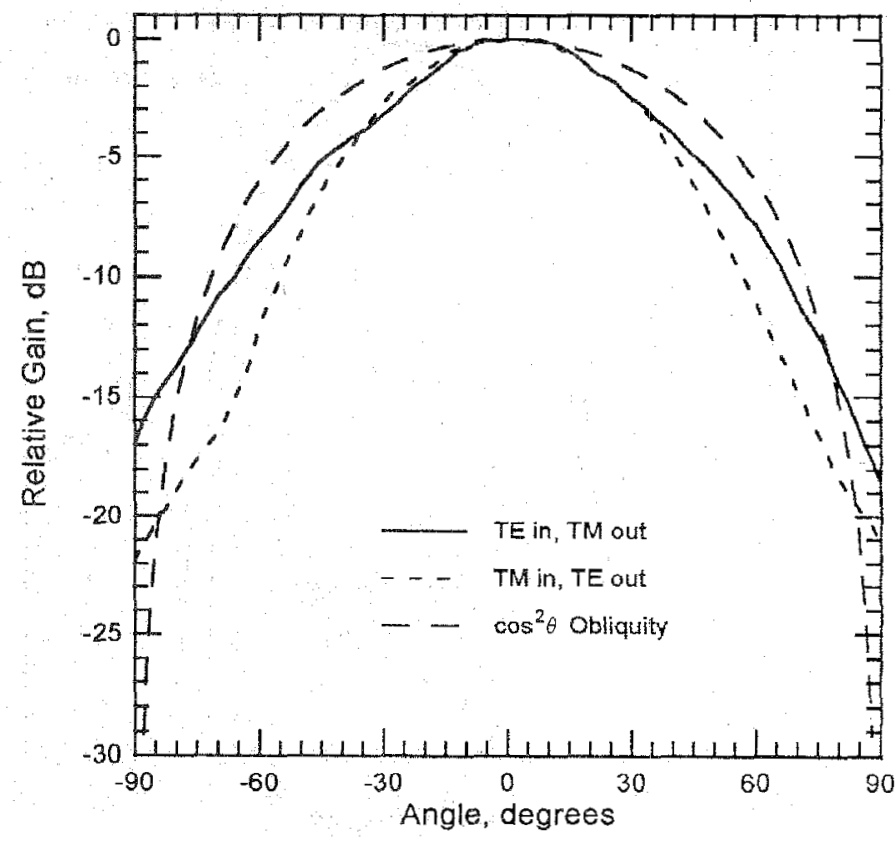

Fig. 14. Relative amplifier gain at $10 \mathrm{GHz}$ as a function of angle with the input polarizer moved closer by one-half wavelength. The peak gain is reduced by $3 \mathrm{~dB}$.

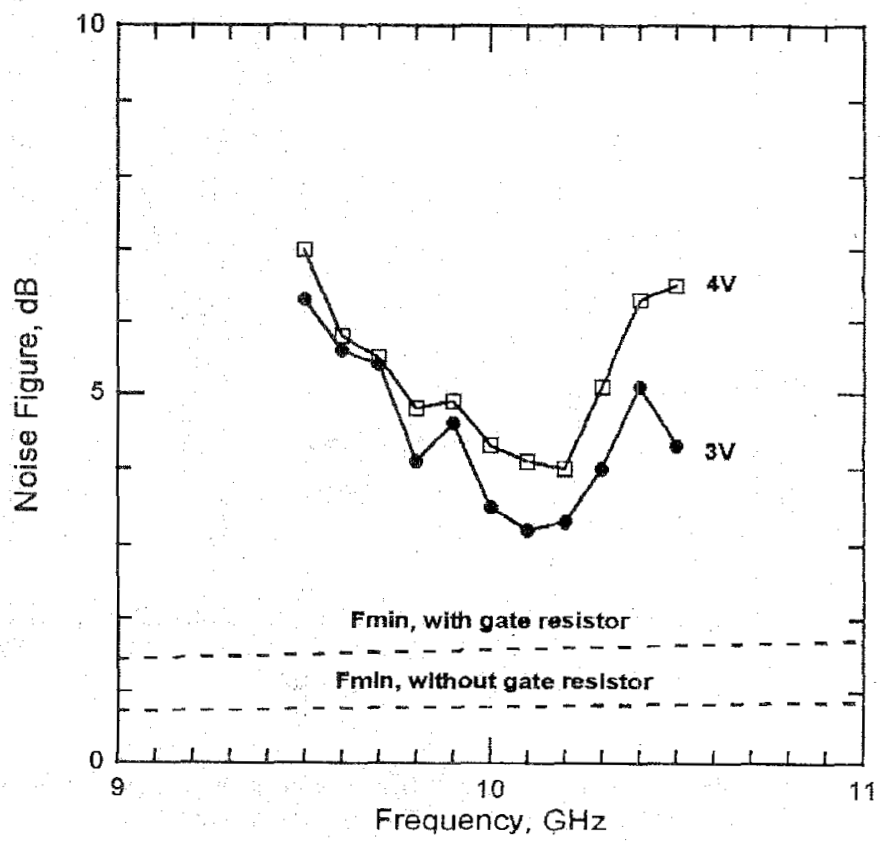

Fig. 15. Measured noise figure at two different bias points.

was $24.5 \mathrm{~W}$. To avoid overheating, we only biased the grid for several seconds at a time.

Fig. 17 shows the power-added efficiency for the grid biased at $3 \mathrm{~V}$ and $7 \mathrm{~V}$. The peak efficiency is $12 \%$. This is more than a factor of two higher than the HBT grid [9]. Discounting the dc power lost in the $100-\Omega$ source resistors, we can figure the efficiency for the pHEMT alone. The peak device power-added efficiency is $20 \%$ for the $3 \mathrm{~V}$ bias at an output power of $1 \mathrm{~W}$.

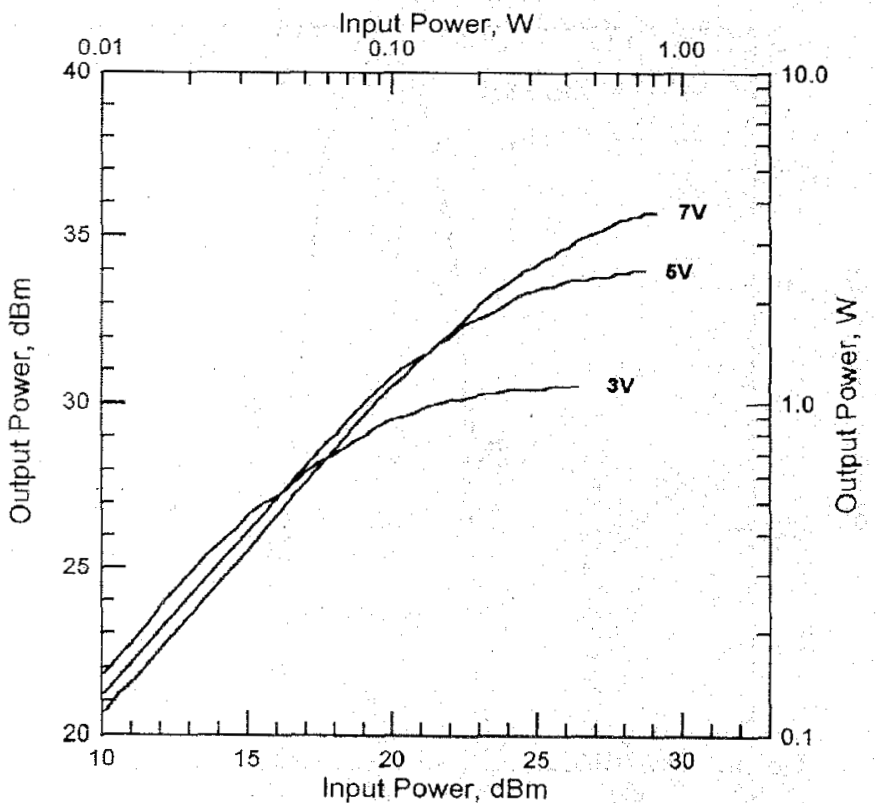

Fig. 16. Output power of the amplifier grid at $9 \mathrm{GHz}$.

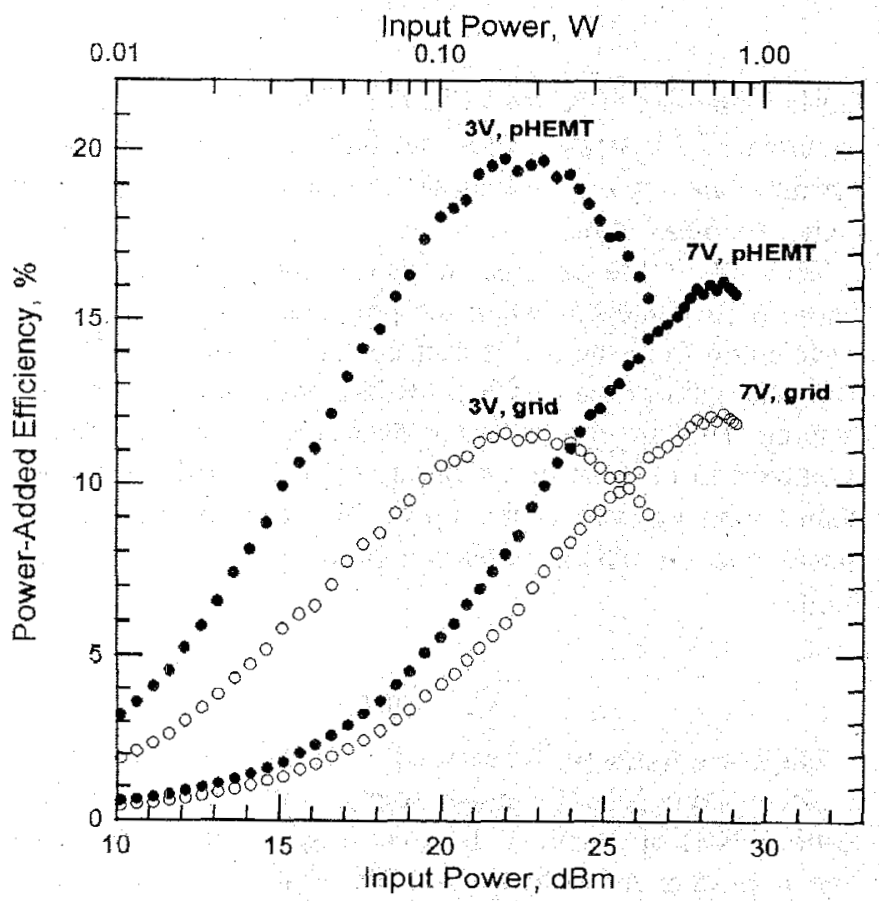

Fig. 17. Power-added efficiency for the amplifier grid at $9 \mathrm{GHz}$. The solid circles are the efficiency for the pHEMT alone, discounting the de power lost in the $100-\Omega$ source resistors.

\section{CONCLUSTON}

We have demonstrated a 100 -element $X$ band pHEMT grid amplifier. The grid has a gain of $10 \mathrm{~dB}$ at $10 \mathrm{GHz}$, and 12 $\mathrm{dB}$ at $9 \mathrm{GHz}$ with a $15 \%$ bandwidth. The minimum noise figure is $3 \mathrm{~dB}$ at $10 \mathrm{GHz}$. The maximum output power is 
$3.7 \mathrm{~W}$ at $9 \mathrm{GHz}$, with a power-added efficiency of $12 \%$. The grid will amplify beams with incidence angles up to $25^{\circ}$. We have also presented a transmission-line model for the grid that predicts gain at normal and oblique incidence with input and output tuning. Furthermore, we have developed a stability model that is useful for suppressing common-mode oscillations.

\section{ACKNOWLEDGMENT}

The authors are grateful to $S$. Weinreb of the University of Massachusetts and N. Byer of Sanders, a Lockheed Martin Company, for their support and suggestions.

\section{REFERENCES}

[1] M. Kim, J. J. Rosenberg, R. P. Smith, R. M. Weikle, J. B. Hacker, M. P. De Lisio, and D. B. Rutledge, "A grid amplifier," IEEE Microwave Guided Wave Lett., vol. 1, pp. 322-324, Nov. 1991

[2] T. Mader, J. Schoenbęrg, L. Harmon, and Z. B. Popović, "Planar MESFET transmission wave amplifier," Electron, Lett., vol. 29, pp. 1699-1701, Sept. 1993.

[3] N. Sheth, T. Ivanov, A. Balasubramaniyan, and A. Mortazawi, "A Nine HEMT Spatial Amplifier," in 1994 IEEE MTT-S Int. Microwave Symp. Dig., 1994, pp. 1239-1242.

[4] J. Schoenberg, T. Mader, B. Shaw, and Z.B. Popović, "Quasi-optical antenna array amplifiers," in 1995 IEEE MTT-S Int. Microwave Symp. Dig., 1995, pp. 605-608.

[5] C.-Y. Chi and G. Rebeiz, "A quasi-optical amplifier," IEEE Microwave Guided Wave Lett., vol. 3, pp. 164-166, June 1993.

[6] T. P. Budka, M. W. Trippe, S. Weinreb, and G. M. Rebeiz, "A $75 \mathrm{GHz}$ to $115 \mathrm{GHz}$ quasi-optical amplifier," IEEE Trans. Microwave Theory Tech., vol. 42, pp. 899-901, May 1994.

[7] H. S. Tsai, M. J. W. Rodwell, and R. A. York, "Planar amplifier array with improved bandwidth using folded-slots," IEEE Microwave Guided Wave Lett., vol. 4, pp. 112-114, Apr. 1994.

[8] N. J. Kolias and R. C. Compton, "A microstrip-based unit cell for quasioptical amplifier arrays," IEEE Microwave Guided Wave Lett., vol. 3, pp. 330-332, Sept. 1993.

[9] M. Kim, E. A. Sovero, J. B. Hacker, M. P. De Lisio, J.-C. Chiao, S.-J. Li, D. R. Gagnon, J. J. Rosenberg, and D. B. Rutledge, "A 100-element HBT grid amplifier," IEEE Trans. Microwave Theory Tech., vol. 41, pp. 1762-1771, Oct. 1993.

[10] D.-W. Tu, S.W. Duncan, A. Eskandarian, B. Golja, B. C. Kane, S. P. Svenson, S. Weinreb, and N. E. Byer, "High gain monolithic W-band low noise amplifiers based on pseudomorphic high electron mobility transistors," IEEE Trans. Microwave Theory Tech., vol. 42, pp. 2590-2597, Dec. 1994

[11] P. M. Smith, D. W. Ferguson, W. F. Kopp, P. C. Chao, W. Hu, P. Ho, and J. M. Ballingall, "A high power, high efficiency millimeter-wave pseudomorphic HEMT," in 1991 IEEE MTT-S Int. Microwave Symp. Dig., 1991, pp. 717-720.

[12] S. Shanfield, A. Platzker, L. Aucoin, T. Kazior, B. I. Patel, A. Bertand, W. Hoke, and P. Lyman, "One-watt, very high efficiency 10 and 18 GHz pseudomorphic HEMT's fabricated by dry first recess etching," in 1992 IEEE MTT-S Int. Microwave Symp. Dig., 1992, pp. 639-641.

[13] M.P. De Lisio, C.-M. Liu, A. Moussessian, D. B. Rutledge, and J. J. Rosenberg, "A 100-element MODFET grid amplifier," in 1995 IEEE AP-S Int. Symp. Dig., 1995, pp. 1304-1307.

[14] C.-M. Liu, E. A. Sovero, M. P. De Lisio, A. Moussessian, J. J. Rosenberg, and D. B. Rutledge, "Gain and stability models for HBT grid amplifiers," in 1995 IEEE AP-S Int. Symp. Dig., 1995, pp. 1292-1295.

[15] M. P. De Lisio, "Hybrid and monolithic active quasi-optical grids," Ph.D. thesis, California Institute of Technology, Pasadena, CA, 1996.

[16] R. D. Martinez and R. C. Compton, "A general approach for the Sparameter design of oscillators with 1 and 2-port active devices," IEEE Trans. Microwave Theory Tech., vol. 40, pp. 569-574, Mar. 1992.

[17] J. Bae, J.-C. Chiao, K. Mizuno, and D. B. Rutledge, "Metal mesh couplers using evanescent wave at millimeter and submillimeter wavelengths," in 1995 IEEE MTT-S Int. Symp. Dig., 1995, pp. 597-600.

[18] H. Fukui, "Optimal noise figure of microwave GaAs MESFET's," IEEE Trans. Electron Devices, vol. 26, pp. 1032-1037, July, 1979.

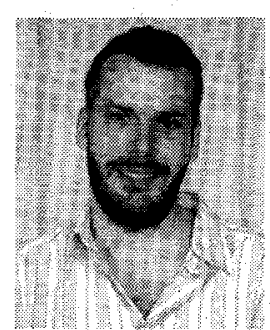

Michael P. De Lisio ( $S^{\prime} 90-M^{\prime} 96$ ) was born in Southfield, MI, on July 29,1968 . He received the B.S.E. degree in electrical engineering from the University of Michigan, Ann Arbor, in 1990, and the M.S. and Ph.D. degrees in electrical engineering from the California Institute of Technology, Pasadena, in 1991, and 1996, respectively.

In January, 1996 he joined the Department of Electrical Engineering, University of HawaiiMānoa, Honolulu, as an Assistant Professor . His research interests include high-frequency solid-state devices, microwave and millimeter-wave power combining, and monolithic quasioptical devices.

Dr. De Lisio was a National Science Foundation Graduate Research Fellow and is a member of Tau Beta Pi and Eta Kappa Nu.

Scott W. Duncan (M'79) received the B.S. degree in physics from Know College, Galesburg, IL, in 1973, and the M.A. and Ph.D. degrees from The Johns Hopkins University, Baltimore, MD, in 1975, and 1978, respectively.

From 1978 to 1981 , he worked on developing magnetic recording heads for disk drives for Semi-Films Inc. In 1981, he joined Martin Marietta Laboratories, Baltimore, MD, where initially he worked on discrete GaAs devices and sub-micron Si processes. From 1985 to 1995 he was in charge of the GaAs MMIC fabrication effort in Baltimore. He has developed MMIC processes for mm-wave switches using $\mathrm{p}$-i-n diodes, downconverters using Schottky mixer diodes, phase shifters and frequency doubles using varactor diodes, and low-noise amplifiers and power amplifiers using pseudomorphic HEMT's. In 1995 he transferred to Sanders, Nashua, NH, a Lockheed Martin Company, where he is currently working to re-establish the processes developed in Baltimore.

Der-Wei Tu received the B.S. degree in electronics engineering from National Chiao Tung university, Hsinchu, Taiwan, R.O.C., in 1976, and the M.A. and Ph.D. degrees in electrical engineering from Princeton University, Princeton, NJ, in 1982 and 1986, respectively.

He joined the University of Maryland, College Park, in 1996 as a Senior Researcher, Department of Electrical Engineering, after 10 years at Martin Marietta Laboratories, Baltimore, MD. While at Martin Marietta, he was a Senior Scientist responsible for advanced device research and process development of $0.1-\mu \mathrm{m}$ pHEMT-based millimeter wave monolithic integrated circuits. He has over 30 publications and conference presentations.

Dr. Tu is a member of Phi Kappa Phi.

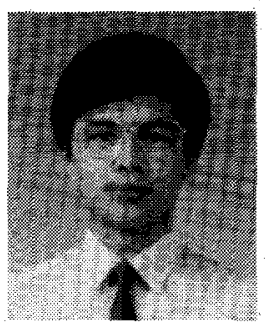

Cheh-Ming Liu was born in Tounan, Taiwan, R.O.C., on August 28,1964 . He received the B.S. and M.S. degrees in electronic engineering from National Chiao-Tung University, Hsinchu, Taiwan, in 1986 and 1988, respectively, and the M.S. and $P h . D$. degrees in electrical engineering from the California Institute of Technology, Pasadena, in 1994 and 1996, respectively.

He joined the Science Center, Rockwell International, Thousand Oaks, CA, as a Member of the Technical Staff in 1996. His research interests include microwave and millimeter-wave grid amplifiers, quasioptical measurement, and computer-aided design. 


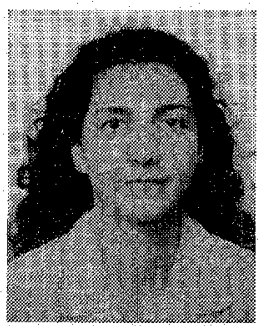

Alina Moussessian was born in Tehran, Iran, on June 21, 1966. She received the B.S: degree in electrical engineering from Iran University of Science and Technology, Tehran, in 1988. In 1992, she received the M.S. degree in electrical engineering. from the California Institute of Technology, Pasadena. She is currently a candidate for the Ph.D. degree at Caltech.

Her research interests include microwave, millimeter-wave, and submillimeter-wave power design and measurement of microwave circuits. combining, beam steering, and computer-aided

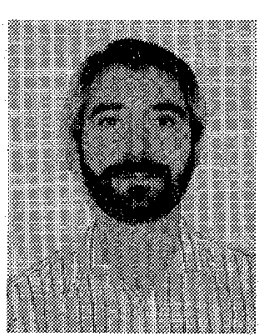

James J. Rosenberg received the Sc.B. degree in engineering from Brown University, Providence, RI, in 1979, the M.S. degree in electrical engineering from the University of California, Berkeley, in 1980, and the Ph.D. degree from Columbia University, New York, NY, in 1983.

He was an Assistant and Associate Professor of Engineering, Brown University, from 1983 to 1990. He was the Deputy Manager of the Microdevices Laboratory, NASA Jet Propulsion Laboratory, Pasadena, CA, during 1990 and 1991. He then served as the Director of Engineering for the Germanium Power Devices Corporation, a manufacturer of semiconductor devices, in 1991 and 1992. Since 1992, he has been with Harvey Mudd College, Claremont, CA, where he currently holds the rank of Associate Professor. His research interests include semiconductor devices, analog and digital signal processing circuits, microwave amplification systems, and clock synchronization schemes for high-speed digital systems.

Dr. Rosenberg has been awarded the NSF Fellowship for graduate study, an NSF Presidential Young Investigator Award, and the 1988 Technical Analysis Corporation President's Award for Excellence in Teaching. He is a member of Tau Beta Pi.

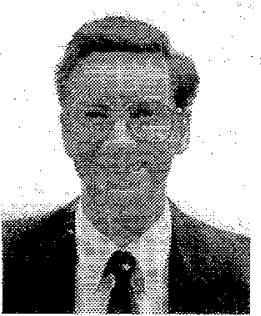

David B. Rutledge is Professor of Electrical Engineering, California Institute of Technology, Pasadena. His research bas been in developing integrated-circuit antennas, imaging arrays, active grids, and software for computer-aided design and measurement. He is co-author with S. Wedge and R. Compton of the widely distributed educational microwave computer-aided design package, Puff with over 14000 copies worldwide. Five of his students have won Presidential investigator and Career Awards. His group has contributed 200 publications to the technical literature.

Dr. Rutledge was Distinguished Lecturè for the Antennas \& Propagation Society and won the Microwave Prize of the Microwave Theory \& Techniques Society and the Teaching Award of the Associated Students of Caltech. 